\title{
ON THE SPATIAL DEGREES OF FREEDOM BENEFITS OF REVERSE TDD IN MULTICELL MIMO NETWORKS
}

\author{
J. Fanjul and I. Santamaria \\ Communications Engineering Dept., University of Cantabria, Santander, Spain \\ e-mail: \{jacobo,nacho\}@gtas.dicom.unican.es
}

\begin{abstract}
In this paper we study the degrees of freedom (DoF) achieved by interference alignment (IA) for cellular networks in reverse time division duplex (R-TDD) mode, a new configuration associated to heterogeneous networks. We derive a necessary feasibility condition for interference alignment in the multi-cell R-TDD scenario, which is then specialized to the particular case of symmetric demands and antenna distribution. We show that, for those symmetric networks for which the properness condition holds with equality, R-TDD does not improve the DoF performance of conventional synchronous TDD systems. Nevertheless, our simulation results indicate that, in more asymmetric scenarios, significant DoF benefits can be achieved by applying the R-TDD approach.
\end{abstract}

Index Terms - Interference alignment, cellular networks, heterogeneous networks, reverse TDD, degrees of freedom.

\section{INTRODUCTION}

Nowadays, the spectral efficiency in multiple-input and multiple-output (MIMO) cellular networks is of paramount importance, due to the exponential growth in terms of wireless data traffic. In this sense, an interesting solution that has attracted considerable amount of research over the last few years is interference alignment (IA), which originated from the degrees of freedom (DoF) analysis of the 2-user X channel [1], [2].

Despite the fact that most of the existing results regarding IA are focused on the well-known interference channel (IC) [3]-[10], there are also remarkable works related to cellular networks. For instance, [11] establishes that a cellular system with $G$ cells and $K$ users per cell, equipped with $N$ and $M$ antennas respectively, is said to be proper if the number of DoF for each link, $d$, satisfies $d \leq \frac{M+N}{G K+1}$, and improper otherwise. As in the case of other network topologies, improper cellular networks are infeasible [12]. However, feasibility of proper cellular networks is still an open area of research.

A set of outer bounds on the DoF for the general $(G, K, M, N)$ model is established in [13], and linear beamforming schemes to achieve IA without symbol extensions are presented in [13]-[15]. Shortly afterwards, authors in [16] identify a genie chain structure and establish the optimality of linear beamforming for some certain regimes.

This work has been supported by the Ministerio de Economía y Competitividad (MINECO) of Spain under grant TEC2013-47141C4-R (RACHEL project) and FPI grant BES-2014-069786.
More recently, standardization groups for next-generation mobile communication have focused on heterogeneous network (HetNet) deployments [17]. In this context, reverse time division duplex (R-TDD) arises as a promising approach, which consists in configuring some cells in uplink mode and the rest of them in downlink simultaneously. Despite works related to interference alignment for these topologies are still scarce, some schemes to achieve IA in cellular networks under R-TDD have already been developed. For instance, the feasibility of IA for R-TDD in 2-cell networks is studied in [18], [19], and authors in [18] establish that the existence of DoF benefits due to the R-TDD approach depends on the network configuration.

In this work, we present a necessary condition for the feasibility of interference alignment in R-TDD multicell networks with arbitrary distribution of users, DoF demands and antennas. Furthermore, we particularize the aforementioned condition to the symmetric DoF and symmetric antenna case. Finally, the DoF performance of the reverse TDD configuration for multicell networks is evaluated via Monte Carlo simulations.

\subsection{Notation}

Uppercase (lowercase) boldface letters will be used for matrices (column vectors), $(\cdot)^{H}$ for conjugate transpose (Hermitian), and $\mathbf{I}_{m, n}$ and $\mathbf{0}_{m, n}$ for the $m \times n$ identity and all-zero matrices, respectively. Additionally, we define the operator

$$
\operatorname{cat}_{s \in \mathcal{S}}\left(\mathbf{A}_{s}\right)
$$

as the horizontal concatenation of the indexed matrices $\mathbf{A}_{s}$ where the members $s$ of the set $S$ are taken in reverse lexicographic order. For instance, consider the set of tuples $\mathcal{S}=\{(1,2),(2,2),(1,1)\} ;$ then

$$
\operatorname{cat}_{s \in \mathcal{S}}\left(\mathbf{A}_{s}\right)=\left[\begin{array}{lll}
\mathbf{A}_{2,2} & \mathbf{A}_{1,2} & \mathbf{A}_{1,1}
\end{array}\right]
$$

Occasionally, we will use $\mathbf{D}(k: \ell, i: j)$ to represent a submatrix of $\mathbf{D}$ consisting of the elements in rows $k$ to $\ell$ and columns $i$ to $j$.

\section{SYSTEM MODEL}

In this work, we consider a MIMO cellular network composed of $G$ cells, where the $g$-th cell contains a base station (BS) and $K_{g}$ user equipments (UE). There is a total of $G_{u}$ uplink cells and $G_{d}$ downlink cells, such that $G=G_{u}+G_{d}$. Each base station is equipped with $N_{g}$ antennas, whereas the $k$-th user 


$$
\mathbf{r}_{k_{g}}=\mathbf{U}_{k_{g}}^{H}(\underbrace{\mathbf{H}_{g, k_{g}} \mathbf{V}_{k_{g}} \mathbf{s}_{k_{g}}}_{\text {desired information }}+\underbrace{\sum_{\substack{\ell_{g}=1 \\ \ell_{g} \neq k_{g}}}^{K_{g}} \mathbf{H}_{g, \ell_{g}} \mathbf{V}_{\ell_{g}} \mathbf{s}_{\ell_{g}}}_{\text {intra-cell interference }}+\underbrace{\sum_{\text {uplink cells }}^{\sum_{j=1}^{G_{u}} \sum_{i_{j}=1}^{K_{j}} \mathbf{H}_{g, i_{j}} \mathbf{V}_{i_{j}} \mathbf{s}_{i_{j}}}+\underbrace{\sum_{j=G_{u}+1}^{G} \sum_{i_{j}=1}^{K_{j}} \mathbf{H}_{g, j} \mathbf{V}_{i_{j}} \mathbf{s}_{i_{j}}}_{\text {downlink cells }}+\mathbf{n}_{g}}_{\text {inter-cell interference }})
$$

$$
\mathbf{r}_{\ell_{j}}=\mathbf{U}_{\ell_{j}}^{H}(\underbrace{\mathbf{H}_{\ell_{j}, j} \mathbf{V}_{\ell_{j}} \mathbf{s}_{\ell_{j}}}_{\text {desired information }}+\underbrace{\sum_{\substack{k_{j}=1 \\ k_{j} \neq \ell_{j}}}^{K_{j}} \mathbf{H}_{\ell_{j}, j} \mathbf{V}_{k_{j}} \mathbf{s}_{k_{j}}}_{\text {intra-cell interference }}+\underbrace{\sum_{\text {uplink cells }}^{\sum_{g=1}^{G_{u}} \sum_{i_{g}=1}^{K_{g}} \mathbf{H}_{\ell_{j}, i_{g}} \mathbf{V}_{i_{g}} \mathbf{s}_{i_{g}}}+\underbrace{\sum_{\substack{g=G_{u}+1 \\ g \neq j}}^{G} \sum_{i_{g}=1}^{K_{g}} \mathbf{H}_{\ell_{j}, g} \mathbf{V}_{i_{g}} \mathbf{s}_{i_{g}}}_{\text {downlink cells }}+\mathbf{n}_{\ell_{j}}}_{\text {inter-cell interference }})
$$

of cell $g$, which we will denote as user $k_{g}$, has $M_{k_{g}}$ antennas, with $g \in\{1,2, \ldots, G\}$ and $k_{g} \in\left\{1,2, \ldots, K_{g}\right\}$.

Since a cellular network in reverse TDD configuration comprises cells in both uplink and downlink mode, we split the notation into signal models (1) and (2), one for each type of cell.

Regarding the uplink cells, base station $g$ applies the decoding matrices $\mathbf{U}_{k_{g}} \in \mathbb{C}^{N_{g} \times d_{k_{g}}}$ to receive $d_{k_{g}}$ independent data streams from each user $k_{g}$, leading to a set of received signals given by (1), where $\mathbf{H}_{g, k_{g}} \in \mathbb{C}^{N_{g} \times M_{k_{g}}}$ represents the channel matrix from user $k_{g}$ to base station $g . \mathbf{s}_{k_{g}} \in \mathbb{C}^{d_{k_{g}} \times 1}$ is the symbol vector transmitted by user $k_{g}$ and precoded by applying the precoding matrix $\mathbf{V}_{k_{g}} \in \mathbb{C}^{M_{k_{g}} \times d_{k_{g}}} . \mathbf{n}_{g} \in \mathbb{C}^{N_{g} \times 1}$ is the additive white Gaussian noise (AWGN) at the input of BS $g$.

Analogously, in the case of downlink cells, the UE $\ell_{j}$ applies a decoder $\mathbf{U}_{\ell_{j}} \in \mathbb{C}^{M_{\ell_{j}} \times d_{\ell_{j}}}$, giving rise to a received signal represented by (2), where $\mathbf{H}_{\ell_{j}, j} \in \mathbb{C}^{M_{\ell_{j}} \times N_{j}}$ is the channel matrix from BS $j$ to $\mathrm{UE} \ell_{j} . \mathbf{s}_{\ell_{j}} \in \mathbb{C}^{d_{\ell_{j}} \times 1}$ denotes the symbol vector transmitted between base station $j$ and user $\ell_{j}$ and precoded by applying the precoder $\mathbf{V}_{\ell_{j}} \in \mathbb{C}^{N_{j} \times d_{\ell_{j}}}$. Finally, $\mathbf{n}_{\ell_{j}} \in \mathbb{C}^{M_{\ell_{j}} \times 1}$ represents the AWGN at the input of user $\ell_{j}$.

Given the received signals in (1) and (2), the interference cancellation conditions can be expressed as ${ }^{1}$

$$
\begin{aligned}
\mathbf{U}_{k_{g}}^{H} \mathbf{H}_{g, i_{j}} \mathbf{V}_{i_{j}}=\mathbf{0}, & \forall k, g, j, i \neq k \\
\mathbf{U}_{g}^{H} \mathbf{H}_{g, j} \mathbf{V}_{i_{j}}=\mathbf{0}, & \forall g, i, j \neq g \\
\mathbf{U}_{\ell_{j}}^{H} \mathbf{H}_{\ell_{j}, i_{g}} \mathbf{V}_{i_{g}}=\mathbf{0}, & \forall \ell, j, i, g \neq j \\
\mathbf{U}_{\ell_{j}}^{H} \mathbf{H}_{\ell_{j}, g} \mathbf{V}_{i_{g}}=\mathbf{0}, & \forall \ell, j, g, i \neq \ell,
\end{aligned}
$$

being $d_{g}=\sum_{k_{g}} d_{k_{g}}$ the total data streams transmitted within

\footnotetext{
${ }^{1}$ For the sake of notation simplicity, the subscript in $\mathbf{0}_{m \times n}$ has been omitted when the dimensions are obvious from the context.
}

the $g$-th cell and $\mathbf{U}_{g} \in \mathbb{C}^{N_{g} \times d_{g}}$ the horizontal concatenation of all $\mathbf{U}_{k_{g}} \in \mathbb{C}^{N_{g} \times d_{k_{g}}}$, i.e.,

$$
\mathbf{U}_{g} \stackrel{\text { def }}{=} \underset{k_{g}}{\operatorname{cat}}\left(\mathbf{U}_{k_{g}}\right)
$$

Condition (3a) corresponds to the interference generated by every UE in uplink mode at the input of a base station in an uplink cell, whereas (3b) is associated to the interference from a base station in downlink mode at the input of a BS in uplink.

Analogously, conditions (3c) and (3d) take into account the interference at a UE in downlink configuration, coming from both users in uplink and base stations in downlink cells, respectively.

Furthermore, we have to guarantee that the DoF of the desired signals are preserved by satisfying the following rank conditions

$$
\begin{array}{r}
\operatorname{rank}\left(\mathbf{U}_{g}^{H}\left[\mathbf{H}_{g, 1_{g}} \mathbf{V}_{1_{g}}, \ldots, \mathbf{H}_{g, K_{g}} \mathbf{V}_{K_{g}}\right]\right)=d_{g}, \quad \forall g \\
\operatorname{rank}\left(\mathbf{U}_{\ell_{j}}^{H} \mathbf{H}_{\ell_{j}, j} \mathbf{V}_{\ell_{j}}\right)=d_{\ell_{j}}, \quad \forall j, \ell_{j} .
\end{array}
$$

Note that conditions (4a) and (4b) refer to the rank preservation for both uplink and downlink cells, respectively.

\section{NECESSARY CONDITION FOR THE FEASIBILITY OF SPATIAL IA}

In this section, we provide a necessary condition for the feasibility of alignment in a general multicell, reverse TDD network. Additionally, we particularize the aforementioned condition for the case of symmetric DoF and equal distribution of antennas, showing that, in some particular cases, R-TDD is not capable of improving the DoF performance of conventional TDD systems. Let us remember that authors in [18] showed that the existence of DoF benefits depends on the network configuration. 
As stated in [19], there is a first condition that needs to be satisfied in every cell within the network:

$$
\sum_{k} d_{k_{g}}=d_{g} \leq N_{g}, \quad \forall g .
$$

Since $\mathbf{U}_{k_{g}}$ and $\mathbf{V}_{k_{g}}$ must be full column rank matrices for all $g, k_{g}$ (see conditions (4a) and (4b)), we can right-multiply them by arbitrary invertible matrices, and both (3) and (4) will still hold. Hence, following the lines established in [20] for X networks and in [19] for 2-cell R-TDD networks, we can rewrite the precoding and decoding matrices as

$$
\mathbf{U}_{k_{g}}=\left[\begin{array}{c}
\mathbf{I}_{k_{k_{g}}} \\
\tilde{\mathbf{U}}_{k_{g}}
\end{array}\right] \mathbf{P}_{k_{g}}^{-1}, \mathbf{V}_{k_{g}}=\left[\begin{array}{c}
\mathbf{I}_{d_{k_{g}}} \\
\tilde{\mathbf{V}}_{k_{g}}
\end{array}\right] \mathbf{Q}_{k_{g}}^{-1} .
$$

where $\mathbf{P}_{k_{g}}=\mathbf{U}_{k_{g}}\left(1: d_{k_{g}},:\right)$ and $\mathbf{Q}_{k_{g}}=\mathbf{V}_{k_{g}}\left(1: d_{k_{g}},:\right)$ are submatrices of $\mathbf{U}_{k_{g}}$ and $\mathbf{V}_{k_{g}}$, respectively.

By transforming the precoders $\mathbf{V}_{k_{g}}$ and decoders $\mathbf{U}_{k_{g}}$ as in $(6), d_{k_{g}}^{2}$ elements are fixed for each precoding/decoding matrix, thus leaving a total of

$$
\begin{aligned}
N_{v} & =\sum_{g=1}^{G_{u}}\left[\left(N_{g}-d_{g}\right) d_{g}+\sum_{k_{g}=1}^{K_{g}}\left(M_{k_{g}}-d_{k_{g}}\right) d_{k_{g}}\right] \\
& +\sum_{g=G_{u}+1}^{G} \sum_{k_{g}=1}^{K_{g}}\left(N_{g}+M_{k_{g}}-2 d_{k_{g}}\right) d_{k_{g}},
\end{aligned}
$$

free variables, where the first line corresponds to the uplink cells and the second term is associated to the downlink cells. Given that, for a system of equations to be proper, the number of equations must be less than or equal to the number of free variables, the inequality

$$
\begin{aligned}
& \sum_{g=G_{u}+1}^{G} \sum_{k_{g}=1}^{K_{g}}\left[d_{k_{g}}\left(\sum_{\substack{\ell_{g}=1 \\
\ell_{g} \neq k_{g}}}^{K_{g}} d_{\ell_{g}}+\sum_{\substack{j=1 \\
j \neq g}}^{G} d_{j}\right)\right] \\
& +\sum_{g=1}^{G_{u}}\left(d_{g} \sum_{\substack{j=1 \\
j \neq g}}^{G} d_{j}\right) \leq N_{v}
\end{aligned}
$$

represents a necessary condition for the feasibility of IA in the considered scenario. Notice that the left side of the inequality in (8) quantifies the total number of scalar equations in the system.

\subsection{Necessary condition for symmetric networks}

Let us consider now a network with symmetric demands and equal distribution of users and antennas for all cells, that is, $d_{k_{g}}=d, K_{g}=K, N_{g}=N, M_{k_{g}}=M \forall g, k_{g}$. Evaluating the expresions above for the number of variables and equations in this case leads to

$$
\begin{aligned}
& N_{v}=K d\left[G_{u}[M+N-(K+1) d]+G_{d}(M+N-2 d)\right], \\
& N_{e}=K d^{2}\left[G_{u} K(G-1)+G_{d}[(K-1)+K(G-1)]\right] .
\end{aligned}
$$

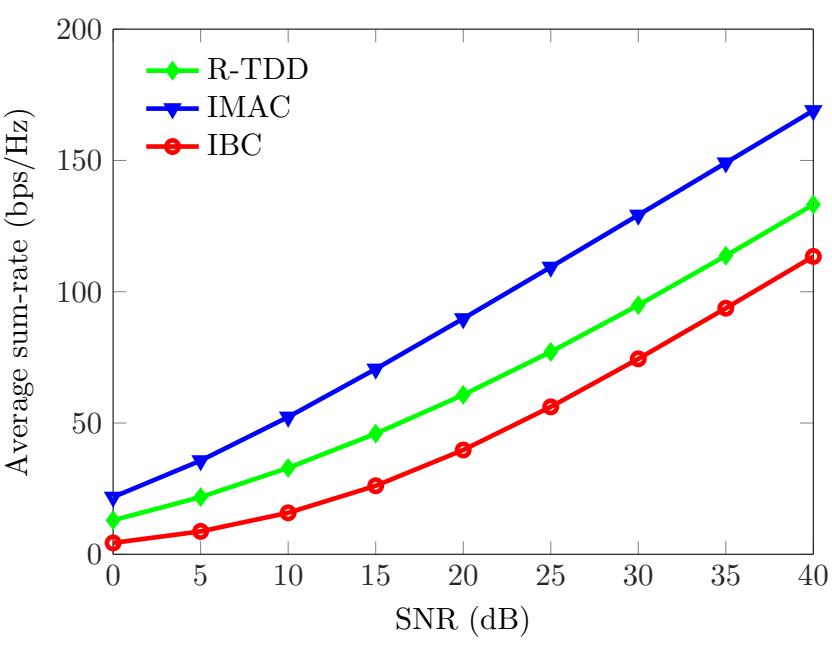

Fig. 1. Average sum-rate achieved by the IBC, IMAC and R-TDD configurations in Network 1.

As in (8), the condition $N_{e} \leq N_{v}$ needs to be satisfied, thus yielding

$$
d \leq \frac{M+N}{G K+1},
$$

which is, in fact, exactly the same condition provided in [11] for conventional TDD systems. Therefore, if (10) holds with equality in a cellular network using a regular TDD configuration, the R-TDD mode will not be able to improve the DoF performance of the system. Nevertheless, there exist many different topologies where reverse TDD yields significant DoF benefits, as originally proved in [18], [19] for 2-cell cases and as we corroborate in this paper for other asymmetric multicell networks.

\section{SIMULATION RESULTS}

In order to analyze the DoF benefits of reverse TDD in multicell networks, we apply the homotopy continuation algorithm in [21] to two different simulation scenarios ${ }^{2}$. For each scenario, the results of 1000 independent trials have been averaged.

\subsection{Symmetric DoF and antenna configuration}

The first network, denoted as Network 1, comprises $G=4$ cells, whose base stations are equipped with $N=10$ antennas. Each cell contains $K=3$ users with $M=3$ antennas each. According to the notation introduced in [19], this is equivalent to a $(10,(3,3,3))^{4}$ MIMO cellular network. For the R-TDD configuration, there are two cells in uplink and two cells in downlink mode.

Figure 1 shows the average sum-rate achieved for the three considered configurations in Network 1, i.e., all cells in uplink or interference multiple-access channel (IMAC), all cells in downlink or interference broadcast channel (IBC), and reverse $\mathrm{TDD}$, as a function of the signal to noise ratio (SNR). Due to

\footnotetext{
${ }^{2}$ As mentioned in [21], cellular networks can be viewed as particular cases of MIMO X networks.
} 


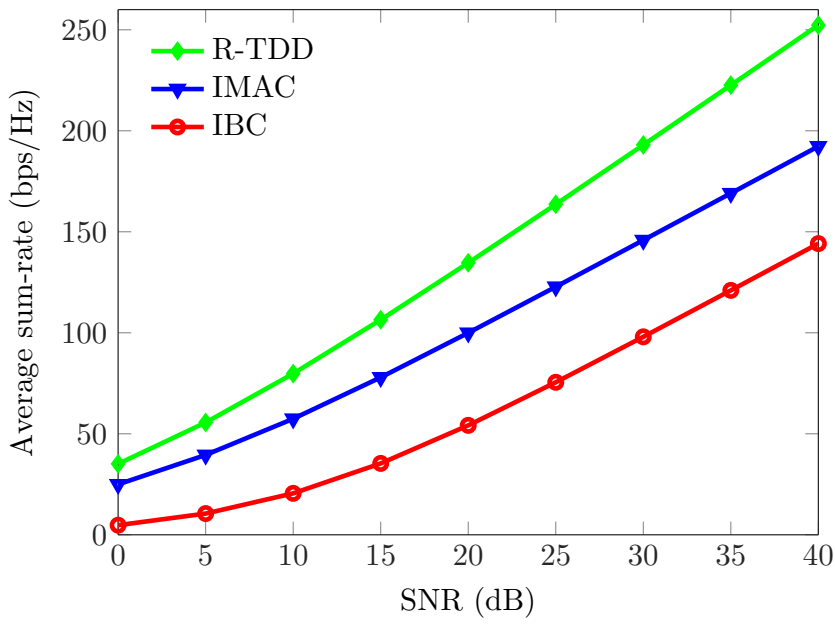

Fig. 2. Average sum-rate achieved by the IBC, IMAC and R-TDD configurations in Network 2.

the duality properties of MIMO networks, both IMAC and IBC will attain the same DoF.

Since Network 1 satisfies the characteristics described in Section 3.1, and as we corroborated in Fig. 1, the sum-rate slope at the high SNR regime is the same for the three operating modes. More specifically, a maximum of 12 interferencefree, independent data streams can be transmitted over this network configuration. This is due to the fact that (10) holds with equality for Network 1 , and consequently the reverse TDD mode is not capable of improving the number of DoF obtained by conventional TDD configurations.

Nevertheless, improvements might be achieved by R-TDD regarding other techniques and figures of merit. For instance, even though the number of DoF does not increase, there might be sum-rate benefits with symmetric distribution of data streams and number of antennas, as shown in [22] for a two-tier R-TDD network.

\subsection{General asymmetric configuration}

We have considered a second example, composed of $G=3$ cells. Specifically, Network 2 is a $(12,(8,8,8,8)) \times(17,(4,4,4))^{2}$ MIMO cellular network, i.e., there is a BS equipped with $N_{1}=12$ antennas, which wants to communicate with $K_{1}=4$ users having $M_{k_{1}}=8$ antennas each. The other two base stations have $N_{2}=N_{3}=17$ antennas and are assigned to $K_{2}=K_{3}=3$ users with $M_{k_{2}}=M_{k_{3}}=4$ antennas each. In the case of the reverse TDD mode, the first cell is in downlink, whereas the remaining cells are configured in uplink. Notice that, for the complementary configuration (i.e., cell 1 in uplink, cells 2 and 3 in downlink), the duality is guaranteed according to $[19]$.

As shown in Fig. 2, R-TDD provides remarkable DoF benefits for this asymmetric network configuration, extending the conclusions of [18], [19] to the multicell context. Specifically, only 14 DoF are obtained by conventional TDD modes, whereas a total of 18 DoF can be achieved by applying R-TDD.

\subsection{Discussion}

In order to provide some insights into the implications of asymmetry in the networks under study, we analyze the configuration of Network 2 in more detail. As mentioned in the previous section, a total of $14 \mathrm{DoF}$ are achieved by applying conventional TDD modes. More specifically, this amount of degrees of freedom is obtained with $d_{k_{1}}=2 \forall k_{1}, d_{k_{2}}=1 \forall k_{2}$ and $d_{k_{3}}=1 \forall k_{3}$, i.e., $d_{1}=8$ and $d_{2}=d_{3}=3$.

In the case of Network 2 in reverse TDD mode, base stations 2 and 3 are configured as receiving nodes, and, since both BS are equipped with much more antennas than the rest of the nodes in the network, these antenna excess could be exploited to align additional interfering streams transmitted from BS 1 . In this case, base station 1 can transmit a total of $d_{1}=12\left(d_{k_{1}}=3\right.$ streams per user $)$, leading to a total of 18 interference-free streams in the network, hence providing a 4 DoF benefit.

When considering the networks described in Section 3.1, for which (10) holds with equality, such antenna excess is not available and hence we cannot choose any cell to support the additional DoF load.

Fortunately, most of the state-of-the-art HetNet topologies comprise, by definition, different tiers including a variety of access points and base stations with different number of users, antennas and communication links. In this context, it is posible to exploit the DoF performance improvements of reverse TDD to its fullest potential.

\section{CONCLUSION}

We have established a necessary condition for the feasibility of interference alignment in MIMO reverse TDD multicell networks. Furthermore, we have specialized the necessary condition to networks with symmetric demands and equal user and antenna distributions, showing that, for those cases where conventional TDD networks attain the properness condition, R-TDD is not capable of achieving a DoF outcome beyond that bound. Our results also show that, for a general R-TDD multicell network, significant benefits can be obtained in terms of maximum degrees of freedom.

\section{References}

[1] S. A. Jafar and S. Shamai (Shitz), "Degrees of Freedom Region of the MIMO X Channel," IEEE Transactions on Information Theory, vol. 54, no. 1, pp. 151-170, Jan. 2008.

[2] M. Maddah-Ali, A. Motahari, and A. Khandani, "Communication over MIMO X Channels: Interference Alignment, Decomposition, and Performance Analysis," IEEE Transactions on Information Theory, vol. 54, no. 8, pp. 3457-3470, Aug. 2008.

[3] V. R. Cadambe and S. A. Jafar, "Interference Alignment and Degrees of Freedom of the K-User Interference Channel," IEEE Transactions on Information Theory, vol. 54, no. 8, pp. 3425-3441, 2008.

[4] S. Peters and R. Heath, "Interference alignment via alternating minimization," in 2009 IEEE International Conference on Acoustics, Speech and Signal Processing (ICASSP), Taipei, Taiwan, Apr. 2009, pp. 2445-2448. 
[5] K. S. Gomadam, V. R. Cadambe, and S. A. Jafar, "A distributed numerical approach to Interference Alignment and applications to wireless interference networks," IEEE Transactions on Information Theory, vol. 57, no. 6, pp. 3309-3322, Jun. 2011.

[6] D. Papailiopoulos and A. Dimakis, "Interference Alignment as a Rank Constrained Rank Minimization," in 2010 IEEE Global Telecommunications Conference (GLOBECOM), Miami, FL, Dec. 2010, pp. 1-6.

[7] M. Razaviyayn, G. Lyubeznik, and Z.-Q. Luo, "On the degrees of freedom achievable through interference alignment in a MIMO interference channel," IEEE Transactions on Signal Processing, vol. 60, no. 2, pp. 812-821, 2012 .

[8] C. Lameiro, Ó. González, and I. Santamaría, "An Interference Alignment Algorithm for Structured Channels," in IEEE 14th Workshop on Signal Processing Advances in Wireless Communications (SPAWC 2013), Darmstadt, Germany, Jun. 2013.

[9] O. González, C. Beltrán, and I. Santamaría, "A Feasibility Test for Linear Interference Alignment in MIMO Channel with Constant Coefficients," IEEE Transactions on Information Theory, vol. 60, no. 3, pp. 18401856, Mar. 2014.

[10] Ó. González, C. Lameiro, and I. Santamaría, "A Quadratically Convergent Method for Interference Alignment in MIMO Interference Channels," IEEE Signal Processing Letters, vol. 21, pp. 1423-1427, Nov. 2014.

[11] B. Zhuang, R. Berry, and M. Honig, "Interference alignment in MIMO cellular networks," in 2011 IEEE International Conference on Acoustics, Speech and Signal Processing (ICASSP), Prague, Czech Republic, May 2011, pp. 3356-3359.

[12] T. Liu and C. Yong, "On the Feasibility of Linear Interference Alignment for MIMO Interference Broadcast Channels With Constant Coefficients," IEEE Transactions on Signal Processing, vol. 61, no. 9, pp. 2178-2191, May 2013.

[13] T. Kim, D. J. Love, and B. Clerckx, "On the Spatial Degrees of Freedom of Multicell and Multiuser MIMO Channels," 2011. arXiv: 1111.3160.

[14] Y. Ma, J. Li, R. Chen, and Q. Liu, "On Feasibility of Interference Alignment for L-Cell Constant Cellular Interfering Networks," IEEE Communication Letters, vol. 16, no. 5, pp. 714-716, May 2012.

[15] G. Sridharan and W. Yu, "Degrees of Freedom of MIMO Cellular Networks: Decomposition and Linear Beamforming Design," IEEE Transactions on Information Theory, vol. 61, no. 6, pp. 3339-3364, Jun. 2015.

[16] T. Liu and C. Yong, "Genie Chain and Degrees of Freedom of Symmetric MIMO Interference Broadcast Channels," 2013. arXiv: 1309.6727.

[17] X. Chu, D. Lopez-Perez, Y. Yang, and F. Gunnarsson, Heterogeneous Cellular Networks. Cambridge, 2013.

[18] S.-W. Jeon and C. Suh, "Degrees of Freedom of UplinkDownlink Multiantenna Cellular Networks," 2014. arXiv: 1404.6012 .
[19] K. Kim, S.-W. Jeon, J. Yang, and D. K. Kim, "The Feasibility of Interference Alignment for Reverse TDD Systems in MIMO Cellular Networks," Oct. 2014. arXiv: 1410.4624 .

[20] H. Sun, C. Geng, T. Gou, and S. A. Jafar, "Degrees of Freedom of MIMO X Networks: spatial scale invariance, one-sided decomposability and linear feasibility," in 2012 IEEE International Symposium on Information Theory Proceedings, Boston, MA, Jul. 2012, pp. 20822086.

[21] Ó. González, J. Fanjul, and I. Santamaría, "Homotopy Continuation for Vector Space Interference Alignment in MIMO X Networks," in 2014 IEEE International Conference on Acoustics, Speech and Signal Processing (ICASSP), May 2014, pp. 6232-6236.

[22] J. Hoydis, K. Hosseini, S. t. Brink, and M. Debbah, "Making smart use of excess antennas: Massive MIMO, Small Cells, and TDD," Bell Labs Technical Journal, vol. 18, no. 2, pp. 5-21, 2013. 